Research Paper

\title{
Elevated plasma level of neutrophil gelatinase-associated lipocalin (NGAL) in patients with breast cancer
}

\author{
Ching-Ting Wei ${ }^{1,8,11,12}$, I-Ting Tsai ${ }^{2,6}$, Cheng-Ching Wu ${ }^{3,6}$, Wei-Chin Hung 3,6 , Chin-Feng Hsuan $3,6,13$, Teng-Hung \\ $\mathrm{Yu}^{3,6}$, Chia-Chang Hsu ${ }^{4,7,14}$, Jer-Yiing Houng ${ }^{9,10}$, Fu-Mei Chung 3 , Yau-Jiunn Lee ${ }^{15}$ and Yung-Chuan Lu ${ }^{5,8}$ \\ 1. Division of General Surgery, Department of Surgery, E-Da Hospital, Kaohsiung, 82445 Taiwan \\ 2. Department of Emergency, E-Da Hospital, Kaohsiung, 82445 Taiwan. \\ 3. Division of Cardiology, Department of Internal Medicine, E-Da Hospital, Kaohsiung, 82445 Taiwan. \\ 4. Division of Gastroenterology and Hepatology, Department of Internal Medicine, E-Da Hospital, Kaohsiung, 82445 Taiwan \\ 5. Division of Endocrinology and Metabolism, Department of Internal Medicine, E-Da Hospital, Kaohsiung, 82445 Taiwan. \\ 6. School of Medicine, College of Medicine, I-Shou University, Kaohsiung, 82445 Taiwan. \\ 7. The School of Chinese Medicine for Post Baccalaureate, College of Medicine, I-Shou University, Kaohsiung, 82445 Taiwan. \\ 8. School of Medicine for International Students, College of Medicine, I-Shou University, Kaohsiung, 82445 Taiwan. \\ . Department of Nutrition, College of Medicine, I-Shou University, Kaohsiung, 82445 Taiwan. \\ 10. Department of Chemical Engineering, I-Shou University, Kaohsiung, 82445 Taiwan. \\ 11. Department of Biomedical Engineering, I-Shou University, Kaohsiung, 82445 Taiwan \\ 12. Department of Electrical Engineering, I-Shou University, Kaohsiung, 82445 Taiwan. \\ 13. Division of Cardiology, Department of Internal Medicine, E-Da Dachang Hospital, Kaohsiung, 80794 Taiwan. \\ 14. Health Examination Center, E-Da Dachang Hospital, Kaohsiung, 80794 Taiwan. \\ 15. Lee's Endocrinology Clinic, Pingtung, 90000 Taiwan. \\ $\triangle$ Corresponding author: Dr. Yung-Chuan Lu, E-Da Hospital, I-Shou University, No. 1, Yi-Da Rd, Jiau-Shu Village, Yan-Chao District, Kaohsiung, 82445, Taiwan. Tel: \\ +886-7-615-1100 ext. 5018; E-mail: ed100369@edah.org.tw.
}

(C) The author(s). This is an open access article distributed under the terms of the Creative Commons Attribution License (https://creativecommons.org/licenses/by/4.0/). See http:/ /ivyspring.com/terms for full terms and conditions.

Received: 2021.01.28; Accepted: 2021.04.27; Published: 2021.05.13

\begin{abstract}
Background: Neutrophil gelatinase-associated lipocalin (NGAL), also known as lipocalin 2, siderocalin, 24p3 or uterocalin, plays a key role in inflammation and in different types of cancer. In this study, we investigated whether plasma NGAL levels were altered in patients with breast cancer. The relationship between plasma NGAL levels and pretreatment hematologic profile was also explored.

Methods: Plasma NGAL concentrations were measured using ELISA in breast cancer patients and control subjects. A total of 75 patients with breast cancer and 65 age- and body mass index-matched control subjects were studied. All of the study subjects were female.

Results: Plasma NGAL level was found to be elevated in the patients with breast cancer compared to the control subjects $(94.3 \mathrm{ng} / \mathrm{mL}$ (interquartile range 39.3-207.6) vs. $55.0 \mathrm{ng} / \mathrm{mL}$ (interquartile range 25.8-124.7), P $=0.007$ ). Multiple logistic regression analysis revealed that NGAL was independently associated with breast cancer, even after adjusting for known biomarkers. Furthermore, NGAL level was elevated in the breast cancer patients who were negative progesterone receptor status, had a histologic grade $\geq 2$, clinical stage III, and pathologic stage T2+T3+T4. In addition, NGAL level was significantly correlated with white blood cell (WBC) count, monocyte count, neutrophil count, and platelet count (all $p<0.01$ ). Moreover, WBC count, neutrophil count, monocyte count, lymphocyte count, platelet count, and NGAL level gradually increased as the stage progressed.

Conclusions: Increased plasma NGAL levels were associated with breast cancer independently of risk factors, and were correlated with inflammatory biomarkers. These results suggest that NGAL may act through inflammatory reactions to play an important role in the pathogenesis of breast cancer.
\end{abstract}

Key words: neutrophil gelatinase-associated lipocalin, breast cancer, white blood cell count, monocyte count, neutrophil count, lymphocyte count, platelet count

\section{Introduction}

Breast cancer is one of the most common cancers in women worldwide. Well-known risk factors for breast cancer include a high body mass index (BMI), increased alcohol consumption, sedentary lifestyle, hormone replacement therapy for menopause, exposure to radiation, early age at menarche, gene 
mutations, and metabolic disorders. In addition, inflammatory cytokines may play an important role [1]. Chronic inflammation has been causally associated with various types of cancer [1-3], and numerous studies have reported that inflammatory infiltrate may be a strong risk factor for cancer development in chronic inflammatory conditions. Lipocalin proteins are involved in inflammation and detoxification processes caused by immune system activation in mammals, and functional roles of lipocalins in human cancers have been determined [4]. Neutrophil gelatinase-associated lipocalin (NGAL), also known as lipocalin 2, siderocalin, 24p3 or uterocalin, is overexpressed in many pathologic conditions, including cancer. NGAL is a $25-\mathrm{kDa}$ glycoprotein that was originally identified as a partner in a covalent complex with neutrophil gelatinase, also known as matrix metalloproteinase 9 (MMP-9) [5,6]. It is frequently associated with tumor stage, size, and invasiveness, and experimental results have demonstrated that NGAL has multiple functions in various cancers, including stimulating proliferation [7], inhibiting apoptosis [8], and promoting the epithelial-to-mesenchymal transition [9]. In addition, the NGAL gene in humans is more highly expressed in luminal epithelial cells than in myoepithelial cells [10], and most breast carcinomas are thought to develop from luminal epithelial cells. Hence, NGAL may actively participate in breast cancer progression [9]. NGAL levels have also been strongly correlated with lymph node metastasis, poor histologic grade, high carcinoma proliferation ability and poor prognosis in breast cancer patients [11].

However, data regarding the role of NGAL and the association between NGAL and hematologic profile in patients with breast cancer are relatively limited. Therefore, the aim of this study was to investigate the role of NGAL in breast cancer. To this end, we measured pretreatment plasma NGAL levels and pretreatment hematologic profiles in a Chinese population with breast cancer.

\section{Methods}

\section{Study participants}

We enrolled 140 Chinese females in this study, including 75 with breast cancer and 65 healthy controls. The breast cancer patients $(n=75)$ were newly diagnosed and had histologically confirmed breast cancer with no prior surgical, chemotherapy, or radiotherapy treatment for breast cancer, and who attended E-Da Hospital between January 2020 and November 2020. The healthy control subjects $(n=65)$ were matched for age and BMI with the breast cancer group. For all subjects, comprehensive questionnaires were used to collect medical information. Complete history was obtained including lifestyle behaviors, medical history, menstrual and reproductive history, and menopausal status, as well as a family history of breast cancer and other cancers. Because the questionnaires were completed prior to undergoing surgery or radio/chemotherapy, any influence of treatment was unlikely. All of the patients and controls who participated in this study were fully informed of the aims of the study and gave written informed consent for their participation. The study protocol was approved by the Human Research Ethics Committee at E-Da Hospital.

\section{Anthropometric measurements and clinical examinations}

Physical and clinical examinations were performed and anthropometric measurements were recorded for all of the participants. Height (to the nearest $0.5 \mathrm{~cm}$ ) and weight (to the nearest $0.1 \mathrm{~kg}$ ) were measured. BMI was calculated as weight in $\mathrm{kg}$ divided by the square of height in meters. Seated blood pressure was also measured by a trained nurse with a digital automatic blood pressure monitor (Omron model HEM-907, Omron, Japan) after the patients had rested for 5 minutes. All of the control subjects were confirmed to be free from benign or malignant breast diseases through physical examinations and mammography. Women with a history or family history of any tumor or cancer were excluded. The diagnosis of breast cancer was confirmed histologically in each case, and estrogen receptor status was determined. The staging of breast cancer was determined according to the TNM system.

\section{Laboratory measurements}

Plasma biochemical parameters were measured after overnight fasting including triglycerides, total cholesterol, creatinine, and glucose, which were measured using standard commercial methods on a parallel, multichannel analyzer (Hitachi7170A, Tokyo, Japan) as in our recent report [12]. Peripheral complete blood cell count was determined using an automated cell counter (XE-2100 Hematology Alpha Transportation System, Sysmex Corporation, Kobe, Japan). To minimize the confounding effect of infection, subjects with a white blood cell (WBC) count below $4.0 \times 10^{9} / 1$ or greater than $10.0 \times 10^{9} / 1$ were re-checked and studied extensively to rule out the existence of chronic infections on the basis of physicians interview, physical examination, and urinalysis.

\section{Plasma NGAL measurements}

All blood samples were drawn after overnight fasting and plasma samples were kept at $-80{ }^{\circ} \mathrm{C}$ for 
subsequent assay. The concentrations of plasma NGAL were determined using commercial enzyme immunoassay kits (Cloud-Clone Corp., Katy, USA). The intraassay coefficient of variation was $<10 \%$ for NGAL. ELISA was performed according to the instructions of the manufacturer, who stated that the assay had excellent specificity for the detection of human NGAL, and no significant cross-reactivity or interference with analogues was observed. Samples were measured in duplicate in a single experiment.

\section{Statistical analysis}

Data normality was analyzed using the Kolmogorov-Smirnov test. Continuous, normally distributed variables are presented as mean $\pm S D$, and non-normally distributed variables as median (interquartile range [IQR]). Statistical differences in the variables were compared using unpaired Student's $t$-tests or Wilcoxon rank sum test (as indicated) for continuous variables. One-way analysis of variance was performed to examine the effect of each variable among the tumor stage groups. Since the distributions of serum triglycerides and plasma NGAL were skewed, logarithmically transformed values were used for the statistical analysis. These variables were assessed for independent associations with the presence of breast cancer in multiple logistic regression analysis using the control subjects as the reference category. Spearman rank correlation coefficients were used to examine correlations between plasma NGAL and the values of other parameters. All of the statistical analyses were two-sided, and a $p$ value $<0.05$ was considered to be significant. All analyses were performed using SAS statistical software, version 8.2 (SAS Institute Inc., Cary, NC, USA).

\section{Results}

Among 106 consecutive breast cancer patients, 31 were excluded due to the following reasons: twenty-one patients refused to join the study after surgery, and 10 patients could not be provided complete information regarding demographic history, biochemical data, and medication treatment. The final study population included 75 patients.

\section{Clinical and biochemical characteristics of study subjects}

Table 1 shows the baseline clinical and biochemical data of the study participants with and without breast cancer. Compared to the healthy control subjects, plasma levels of NGAL were significantly higher among those with breast cancer $(55.0 \mathrm{ng} / \mathrm{ml}$ (IQR: $25.8-124.7)$ versus $94.3 \mathrm{ng} / \mathrm{ml}$ (IQR: 39.3-207.6, $p=0.007)$. Furthermore, the patients with breast cancer had higher fasting glucose, WBC count, neutrophil count, monocyte count, and platelet count than the healthy control subjects. The mean age, BMI, blood pressure, total cholesterol, triglycerides, serum glutamate oxaloacetate transaminase, serum glutamic pyruvic transaminase, creatinine, estimated glomerular filtration rate, albumin, lymphocyte, red blood cells, hemoglobin, and hematocrit were similar in both groups.

\section{Associations between plasma NGAL and breast cancer}

Multivariate logistic regression analysis was performed to estimate the effects of plasma NGAL level together with other breast cancer risk factors on the presence of breast cancer. The results showed that the presence of breast cancer was associated with BMI, fasting glucose, neutrophil count, and NGAL level (Table 2).

Table 1. Baseline clinical and biochemical characteristics of the study population

\begin{tabular}{|c|c|c|c|}
\hline Parameter & Breast cancer & Healthy controls & $p$-value \\
\hline No. & 75 & 65 & \\
\hline Age (years) & $55.3 \pm 11.2$ & $56.0 \pm 7.1$ & 0.702 \\
\hline Body mass index $\left(\mathrm{kg} / \mathrm{m}^{2}\right)$ & $25.5 \pm 4.0$ & $24.3 \pm 4.4$ & 0.103 \\
\hline Systolic blood pressure (mmHg) & $131 \pm 18$ & $125 \pm 18$ & 0.062 \\
\hline Diastolic blood pressure ( $\mathrm{mmHg}$ ) & $77 \pm 10$ & $77 \pm 13$ & 0.702 \\
\hline Fasting glucose $(\mathrm{mg} / \mathrm{dl})$ & $127.9 \pm 48.9$ & $99.8 \pm 21.6$ & 0.0001 \\
\hline Total cholesterol (mg/dl) & $189.8 \pm 33.6$ & $192.8 \pm 39.5$ & 0.752 \\
\hline Triglycerides (mg/dl) & $136(69-214)$ & $88(66-122)$ & 0.177 \\
\hline SGOT (U/L) & $28.9 \pm 19.4$ & $29.3 \pm 9.6$ & 0.910 \\
\hline SGPT (U/L) & $26.8 \pm 22.8$ & $28.1 \pm 15.9$ & 0.704 \\
\hline Creatinine (mg/dl) & $0.95 \pm 0.18$ & $0.93 \pm 0.09$ & 0.459 \\
\hline Estimated GFR $\left(\mathrm{ml} / \mathrm{min} / 1.73 \mathrm{~m}^{2}\right)$ & $93.7 \pm 19.2$ & $93.1 \pm 12.8$ & 0.839 \\
\hline Albumin (g/dl) & $4.3 \pm 0.3$ & $4.2 \pm 0.3$ & 0.586 \\
\hline White blood cell count $\left(10^{9} / 1\right)$ & $6.808 \pm 2.758$ & $5.904 \pm 1.694$ & 0.029 \\
\hline Neutrophil count $\left(10^{9} / 1\right)$ & $4488 \pm 2653$ & $3389 \pm 1333$ & 0.006 \\
\hline Monocyte count $\left(10^{9} / 1\right)$ & $426 \pm 212$ & $296 \pm 101$ & $<0.0001$ \\
\hline Lymphocyte count $\left(10^{9} / 1\right)$ & $1790 \pm 559$ & $1993 \pm 639$ & 0.069 \\
\hline Red blood cells $\left(\times 10^{6} / \mu \mathrm{l}\right)$ & $4.52 \pm 0.53$ & $4.42 \pm 0.32$ & 0.199 \\
\hline Hemoglobin (g/dL) & $12.6 \pm 1.7$ & $12.8 \pm 1.0$ & 0.379 \\
\hline Hematocrit (\%) & $38.1 \pm 5.3$ & $38.6 \pm 2.5$ & 0.475 \\
\hline Platelet count $\left(\times 10^{3} / \mu \mathrm{l}\right)$ & $280.6 \pm 82.9$ & $249.4 \pm 54.9$ & 0.014 \\
\hline NGAL (ng/ml) & $94.3(39.3-207.6)$ & $55.0(25.8-124.7)$ & 0.007 \\
\hline
\end{tabular}

Data are mean \pm SD or median (interquartile range). SGOT, serum glutamate oxaloacetate transaminase; SGPT, serum glutamic pyruvic transaminase; GFR, glomerular filtration rate, NGAL, neutrophil gelatinase-associated lipocalin.

\section{Association between NGAL level and clinicopathologic markers}

NGAL level was elevated in the breast cancer patients who were progesterone receptor (PR) negative, had a histologic grade $\geq 2$, clinical stage III, and pathologic stage $\mathrm{T} 2+\mathrm{T} 3+\mathrm{T} 4$. However, there were no significant associations between estrogen receptor (ER) status (positive: negative), HER-2 receptor status (positive: negative), tumor size $(\leq 1:>1)$, and lymph 
node metastasis $(\mathrm{N} 0+\mathrm{N} 1: \mathrm{N} 2+\mathrm{N} 3)$ and plasma NGAL level (all p > 0.05).

Table 2. Multiple logistic regression analysis with the presence of breast cancer as the dependent variable

\begin{tabular}{llll}
\hline & $\exp (\mathrm{B})$ & $95 \%$ confidence interval & $p$-value \\
\hline Age & 0.87 & $0.73-1.03$ & 0.105 \\
Body mass index & 1.38 & $1.04-1.84$ & 0.028 \\
SBP & 1.08 & $0.99-1.18$ & 0.090 \\
DBP & 0.84 & $0.72-0.98$ & 0.052 \\
Total cholesterol & 1.01 & $0.98-1.05$ & 0.496 \\
Triglycerides & 1.00 & $0.99-1.02$ & 0.898 \\
Fasting glucose & 1.07 & $1.01-1.12$ & 0.012 \\
SGOT & 1.20 & $0.99-1.47$ & 0.068 \\
SGPT & 0.90 & $0.78-1.03$ & 0.121 \\
Estimated GFR & 0.96 & $0.88-1.05$ & 0.404 \\
White blood cell count & 0.67 & $0.36-1.25$ & 0.205 \\
Neutrophil count & 1.19 & $1.01-1.39$ & 0.033 \\
Monocyte count & 2.04 & $0.84-4.93$ & 0.113 \\
Platelet count & 1.02 & $0.99-1.03$ & 0.079 \\
NGAL & 1.01 & $1.00-1.02$ & 0.040 \\
\hline
\end{tabular}

SBP, systolic blood pressure; DBP, diastolic blood pressure; SGOT, serum

glutamate oxaloacetate transaminase; SGPT, serum glutamic pyruvic transaminase;

GFR, glomerular filtration rate; NGAL, neutrophil gelatinase-associated lipocalin.

\section{Correlations among NGAL level and pretreatment hematologic parameters}

Spearman rank correlation analysis showed that plasma NGAL levels were correlated with WBC count, monocyte count, neutrophil count, and platelet count (Table 4). Linear contrast analysis was conducted to evaluate the correlations among WBC count, neutrophil count, monocyte count, lymphocyte count, platelet count, NGAL level, and tumor stage (Figure 1). The results showed that WBC count, neutrophil count, monocyte count, lymphocyte count, platelet count, and NGAL levels gradually increased as the stage progressed $(p<0.01)$.

\section{Discussion}

In this study, we demonstrated that plasma NGAL concentrations were significantly elevated in a fully adjusted model in patients with breast cancer.
Furthermore, the level of NGAL gradually increased as the stage progressed, along with elevations in PR-negative status, histologic grade $\geq 2$, clinical stage III, and pathologic T2+T3+T4. Moreover, we found significant correlations between plasma NGAL concentrations and WBC count, monocyte count, neutrophil count, and platelet count. To the best of our knowledge, this is the first report to describe elevated NGAL plasma concentrations in patients with breast cancer.

Table 3. Median (interquartile range) baseline plasma concentration of neutrophil gelatinase-associated lipocalin grouped according to clinicopathologic marker status

\begin{tabular}{|c|c|c|c|}
\hline Parameters & $\mathrm{N}$ & NGAL (ng/ml) & $p$-value \\
\hline \multicolumn{4}{|c|}{ Estrogen receptor status } \\
\hline Positive & 62 & $72.6(27.2-137.9)$ & 0.198 \\
\hline Negative & 13 & $97.2(42.6-213.2)$ & \\
\hline \multicolumn{4}{|c|}{ Progesterone receptor status } \\
\hline Positive & 53 & $64.5(33.3-115.9)$ & 0.016 \\
\hline Negative & 22 & $104.8(45.5-226.2)$ & \\
\hline \multicolumn{4}{|c|}{ HER-2 receptor status } \\
\hline Positive & 60 & $100.6(44.2-213.2)$ & 0.070 \\
\hline Negative & 15 & $72.2(26.9-122.1)$ & \\
\hline \multicolumn{4}{|c|}{ Histologic grade } \\
\hline 1 & 25 & $61.4(31.1-101.8)$ & 0.006 \\
\hline$\geq 2$ & 50 & $132.9(44.5-228.3)$ & \\
\hline \multicolumn{4}{|c|}{ Tumor size $(\mathrm{cm})$} \\
\hline$\leq 1$ & 17 & $72.2(29.6 \pm 166.1)$ & 0.271 \\
\hline$>1$ & 58 & $100.6(43.0 \pm 217.0)$ & \\
\hline \multicolumn{4}{|c|}{ Clinical stage } \\
\hline Stage I & 28 & $57.4(26.8 \pm 182.0)$ & 0.048 \\
\hline Stage II & 31 & $97.4(54.0-218.4)$ & \\
\hline Stage III & 16 & $104.7(47.0-413.6)$ & \\
\hline \multicolumn{4}{|c|}{ Pathologic T stage } \\
\hline $\mathrm{T} 0+\mathrm{T} 1$ & 35 & $81.0(31.0-168.7)$ & 0.029 \\
\hline $\mathrm{T} 2+\mathrm{T} 3+\mathrm{T} 4$ & 40 & $99.1(54.0-227.9)$ & \\
\hline \multicolumn{4}{|c|}{ Lymph node metastasis } \\
\hline $\mathrm{N} 0+\mathrm{N} 1$ & 69 & $90.4(41.3-207.2)$ & 0.149 \\
\hline $\mathrm{N} 2+\mathrm{N} 3$ & 6 & $151.1(23.5-536.9)$ & \\
\hline
\end{tabular}

Table 4. Spearman rank correlation coefficients of the study variables in the patients

\begin{tabular}{|c|c|c|c|c|c|c|c|c|c|c|}
\hline & $\begin{array}{l}\text { WBC } \\
\text { count }\end{array}$ & $\begin{array}{l}\text { Monocyte } \\
\text { count }\end{array}$ & $\begin{array}{l}\text { Neutrophil } \\
\text { count }\end{array}$ & $\begin{array}{l}\text { Lymphocyte } \\
\text { count }\end{array}$ & Albumin & Red blood cells & Hemoglobin & Hematocrit & $\begin{array}{l}\text { Platelet } \\
\text { count }\end{array}$ & NGAL \\
\hline WBC count & 1 & $0.594^{\star *}$ & $0.939^{* *}$ & $0.615^{\star *}$ & -0.083 & $0.258^{* *}$ & 0.135 & 0.106 & $0.343^{* *}$ & $0.307^{* *}$ \\
\hline Monocyte count & & 1 & $0.541^{* *}$ & $0.233^{* *}$ & -0.036 & 0.131 & $0.187^{*}$ & 0.144 & $0.277^{* *}$ & $0.257^{* *}$ \\
\hline Neutrophil count & & & 1 & $0.400^{* *}$ & -0.024 & $0.282^{* * *}$ & 0.121 & 0.114 & $0.403^{* *}$ & $0.357^{* *}$ \\
\hline Lymphocyte count & & & & 1 & -0.136 & 0.130 & $0.246^{\star *}$ & $0.199 *$ & $0.234^{*}$ & 0.122 \\
\hline Albumin & & & & & 1 & $0.318^{*}$ & $0.314^{*}$ & $0.291^{*}$ & 0.188 & 0.233 \\
\hline Red blood cells & & & & & & 1 & $0.391^{\star *}$ & $0.499^{* *}$ & $0.279^{* *}$ & 0.146 \\
\hline Hemoglobin & & & & & & & 1 & $0.919^{* *}$ & 0.080 & -0.049 \\
\hline Hematocrit & & & & & & & & 1 & $0.174^{*}$ & -0.021 \\
\hline Platelet count & & & & & & & & & 1 & $0.296^{* *}$ \\
\hline NGAL & & & & & & & & & & 1 \\
\hline
\end{tabular}



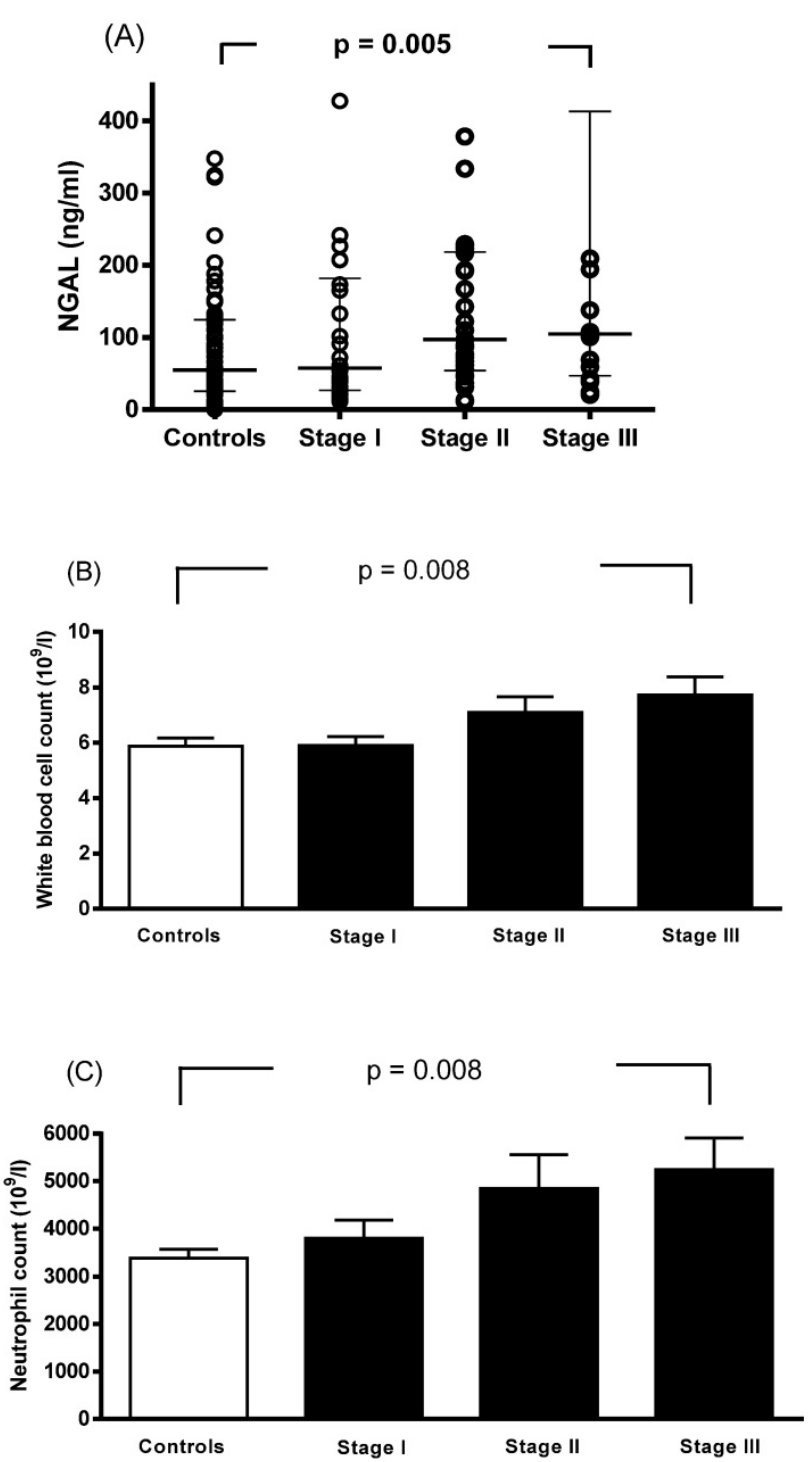

(D)
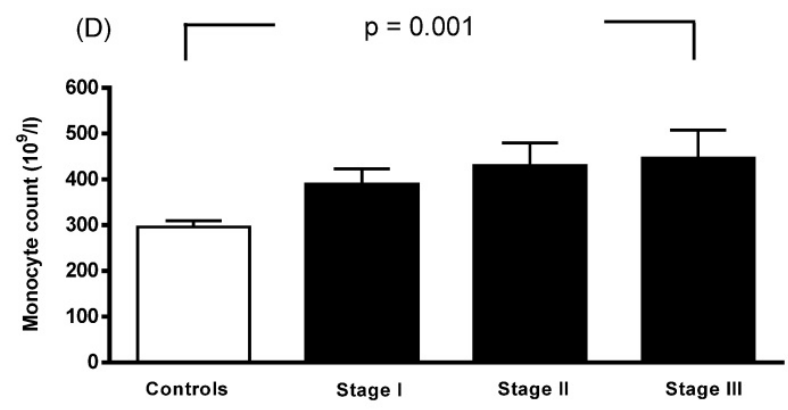

(E)
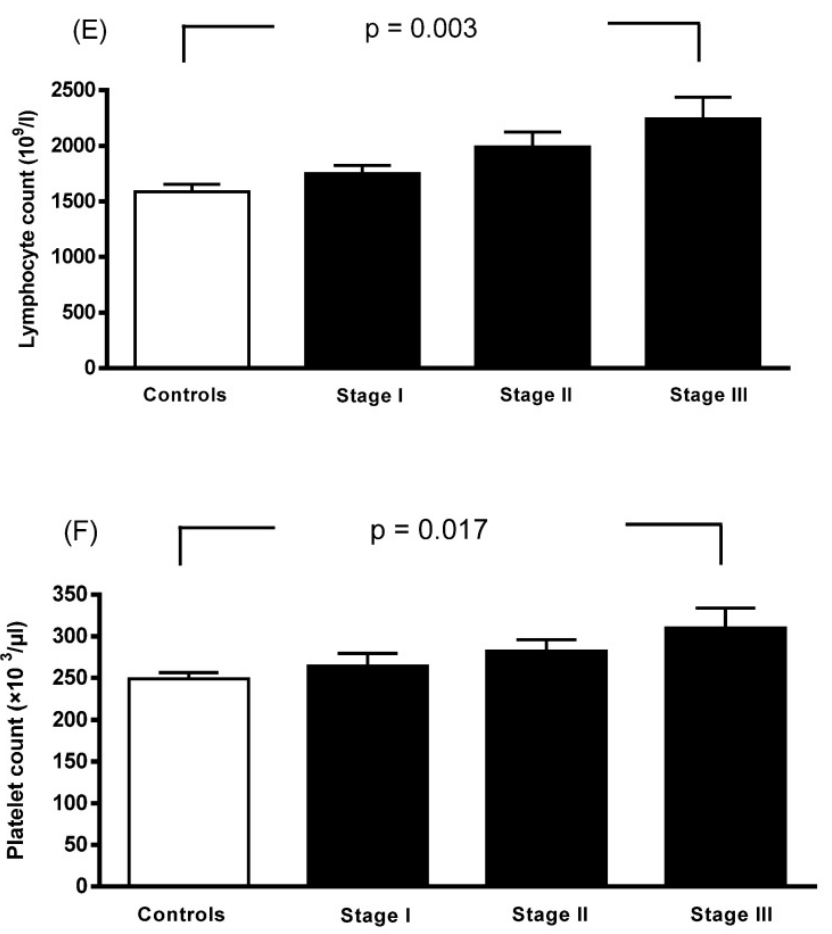

Figure 1. Associations between neutrophil gelatinase-associated lipocalin (A), white blood cell count (B), neutrophil count (C), monocyte count (D), lymphocyte count (E), platelet count $(F)$, and stage progression of breast cancer. Bars represent the mean \pm SD or median (interquartile range). Differences between groups were analyzed using one-way analysis of variance.

Several molecular forms of NGAL have been identified: a disulfide-linked homodimer (46-kDa), a monomer (25-kDa), and a disulfide-linked heterodimer with human neutrophil gelatinase B $(135-\mathrm{kDa})[6,13]$. NGAL is expressed in many other types of cells besides neutrophils. When NGAL is present in a complex with MMP-9, there is less degradation of MMP-9 due to the effects on NGAL on the stability of MMP-9. This results in higher gelatinolytic activity of MMP-9 on the extracellular matrix $[13,14]$. This biological function of NGAL may influence the development of diverse cancer types [14-17]. NGAL is an acute-phase protein that has been implicated in diverse physiological processes in mice, including ion transport, apoptosis, inflammation, cell survival, and tumorigenesis $[18,19]$. NGAL has been shown to promote leukocyte recruitment in the tumor microenvironment through ion-mediated chemokine production [20] and also in activated immune cells such as monocytes and neutrophils [6,21], and the dimer has been shown to be the major molecular form of free NGAL secreted by neutrophils [22]. As NGAL can activate leukocytes, it can be regarded as a pro-inflammatory [20], immunomodulating [23], and apoptosis-inducing cytokine [24]. In the present study, plasma NGAL levels were found to be elevated in the patients with breast cancer, and elevated plasma NGAL levels were associated with total WBC count, monocyte count, and neutrophil count. Based on the findings of previous studies and our data, we propose that NGAL should be considered as a marker of inflammation that participates in the process of breast cancer. 
Chronic inflammatory reactions have been shown in tumors, and they may be associated with cancer progression and chemoresistance. NGAL gets part of its name from neutrophils, as it was determined to be released by neutrophils at sites of infection and inflammation [23]. A previous study found that the expression of NGAL could be up-regulated by interleukin-1beta in a type II pneumocyte-derived cell line through the induction of the nuclear factor-kappaB (NF-kB) pathway [25]. Furthermore, NGAL has been shown to be strongly induced by NF-kB in thyroid carcinomas, an important factor involved both in tumor growth and in the link between chronic inflammation and neoplastic development [26,27]. Hypoxic tumors, defined as tumor growth occurring at a distant region from blood vessels in tumor tissue, have been implicated in tumor metastasis, tumor invasion, and chemotherapy resistance, and are thus considered to be an indicator of tumor malignancy [28-30]. Nakamura et al. demonstrated that NGAL was a useful plasma marker for hypoxic tumors [31]. Furthermore, previous studies have identified that NGAL can actively promote breast cancer metastasis by inducing the production of vascular endothelial growth factor, angiogenesis, epithelial-tomesenchymal transition and cell migration, and invasion through multiple signaling pathways, including phosphoinositide-3-kinase/protein kinase $\mathrm{B} / \mathrm{NF}-\mathrm{kB}$ and hypoxia inducible factor-1a/ extracellular signal-regulated kinase [10,32-34]. Notably, silencing NGAL in breast cancer cells has been shown to reduce tumor progression. Moreover, a few studies have correlated the expression of NGAL to the clinical outcomes of cancer patients $[11,35,36]$. Bauer et al. [11] showed that in primary human breast cancer, NGAL expression was a predictor of poor prognosis, and suggested that the presence of NGAL may assist in risk assessment and identifying a subset of patients requiring more aggressive adjuvant therapy. Hu et al. [35] provided strong evidence for the role of NGAL in aggressive subtypes of breast cancer, metastasis and poor prognosis. Due to its property as a secretory protein, NGAL can be easily detected in the urine or blood circulation, and previous studies have suggested that NGAL may be a prognostic biomarker and noninvasive diagnostic test for breast cancer progression. Wenners et al. [36] also showed that in low-risk subgroups, NGAL was a predictive marker for a pathological complete response after neoadjuvant chemotherapy. Furthermore, NGAL was shown to be an independent prognostic factor for a decrease in disease-free survival in primary human breast cancer. In addition, Venkatesha et al. [37] showed that NGAL represses ras-induced expression of vascular endothelial growth factor in 4T1 cells through the down-regulation of ras mitogen-activated protein kinase and ras phosphatidylinositol-3-kinase signaling. Hence, this study provided new insights into the action of NGAL and raise the possibility that the administration of NGAL may be useful for inhibiting tumor angiogenesis, in addition to suppressing tumor metastasis, in cancers which show ras activation. In the present study, we found that NGAL was associated with breast cancer, and that its concentration gradually increased as the stage progressed in accordance with previous reports $[10,11]$. In addition, our results also showed that NGAL concentration was positively correlated with platelet count. A previous study reported that a high platelet count was associated with metastasis and poor prognosis in cancer patients $[38,39]$. Therefore, the correlation between NGAL and platelet count may explain the increased risk of breast cancer in patients with a high NGAL level.

Stuckey et al. showed that in normal human mammary epithelial cells, the expression of NGAL is controlled by estrogen [40], while in malignant human mammary epithelial cells, NGAL appears to escape from hormonal regulation, since this protein is most abundant in ER-negative breast cancer cell lines and primary tumor samples [11]. Furthermore, Stoesz et al. found that in human breast tumors, the expression of NGAL was not significantly associated with HER-2/neu activation. In contrast, they found significant associations between NGAL expression and several other poor prognostic markers in patients with breast cancer, including ER- and PR-negative status and high proliferation (S-phase fraction) [41]. Interestingly, in the present study, we demonstrated that NGAL was elevated in the patients with PR-negative status, histologic grade $\geq 2$, clinical stage III, and pathologic T2+T3+T4. Hence, our findings suggest that NGAL may be actively contributive to breast cancer progression. However, NGAL was not elevated in the ER-negative patients in the present study, possibly due to the limited sample size.

Some limitations of this study need to be considered. First, our study population was relatively small, and so further studies with larger populations are needed. Moreover, the cross-sectional design limited our ability to infer any causal relationship between NGAL and breast cancer. A prospective cohort study is required to completely elucidate the importance of NGAL as a biomarker of breast cancer and the causative association between breast cancer and the changes in NGAL levels. In addition, although we controlled for other major cancer risk factors, the existence of unrecognized confounding 
variables is always possible. Further investigation is needed to investigate the association between NGAL plasma levels with the prognosis and survival of the breast cancer.

\section{Conclusions}

In conclusion, we found that NGAL plasma concentrations were elevated in patients with breast cancer in our Chinese study population, and that there was possibly a close relationship between NGAL and inflammation and the development of breast cancer. NGAL may be involved in the complex interactions involving inflammatory or immune responses. Further investigations are required to explore the detailed mechanisms of NGAL in the development and progression of breast cancer, and its utility in breast cancer therapy.

\section{Acknowledgements}

The authors would like to thank E-Da Hospital of the Republic of China, Taiwan, for financially supporting this research under contracts EDAHP109001 and EDAHI109002.

\section{Competing Interests}

The authors have declared that no competing interest exists.

\section{References}

1. Esquivel-Velázquez $\mathrm{M}$, Ostoa-Saloma $\mathrm{P}$, Palacios-Arreola MI, Nava-Castro KE, Castro JI, Morales-Montor J. The role of cytokines in breast cancer development and progression. J Interferon Cytokine Res. 2015; 35: 1-16.

2. Coussens LM, Werb Z. Inflammation and cancer. Nature. 2002; 420: 860-7.

3. Mantovani A, Allavena P, Sica A, Balkwill F. Cancer-related inflammation. Nature. 2008; 454: 436-44.

4. Bratt T. Lipocalins and cancer. Biochim Biophys Acta. 2000; 1482: 318-26.

5. Triebel S, Blaser J, Reinke H, Tschesche H. A $25 \mathrm{kDa}$ alpha 2-microglobulin-

related protein is a component of the $125 \mathrm{kDa}$ form of human gelatinase. FEBS Lett. 1992; 314: 386-8.

6. Kjeldsen L, Johnsen AH, Sengelov H, Borregaard N. Isolation and primary structure of NGAL, a novel protein associated with human neutrophil gelatinase. J Biol Chem. 1993; 268:10425-32.

7. Seth P, Porter D, Lahti-Domenici J, Geng Y, Richardson A, Polyak K. Cellular and molecular targets of estrogen in normal human breast tissue. Cancer Res. 2002; 62: 4540-4.

8. Devireddy LR, Gazin C, Zhu X, Green MR. A cell-Surface receptor for lipocalin $24 \mathrm{p} 3$ selectively mediates apoptosis and iron uptake. Cell. 2005; 123: 1293-305.

9. Yang J, Bielenberg DR, Rodig SJ, Doiron R, Clifton MC, Kung AL, et al. Lipocalin 2 promotes breast cancer progression. Proc Natl Acad Sci U S A. 2009; 106: 3913-18.

10. Jones C, Mackay A, Grigoriadis A, Cossu A, Reis-Filho JS, Fulford L, et al. Expression profiling of purified normal human luminal and myoepithelial breast cells: identification of novel prognostic markers for breast cancer. Cancer Res. 2004; 64: 3037-45.

11. Bauer M, Eickhoff JC, Gould MN, Mundhenke C, Maass N, Friedl A. Neutrophil gelatinase-associated lipocalin (NGAL) is a predictor of poor prognosis in human primary breast cancer. Breast Cancer Res Treat. 2008; 108: 389-97.

12. Tsai IT, Wu CC, Hung WC, Lee TL, Hsuan CF, Wei CT, et al. FABP1 and FABP2 as markers of diabetic nephropathy. Int J Med Sci. 2020; 17: $2338-45$.
13. Yan L, Borregaard N, Kjeldsen L, Moses MA. The high molecular weight urinary matrix metalloproteinase (MMP) activity is a complex of gelatinase B/MMP-9 and neutrophil gelatinase-associated lipocalin (NGAL). Modulation of MMP-9 activity by NGAL. J Biol Chem. 2001; 276: 37258-65.

14. Fernández CA, Yan L, Louis G, Yang J, Kutok JL, Moses MA. The matrix metalloproteinase-9/neutrophil gelatin associated lipocalin complex plays a role in breast tumor growth and is present in the urine of breast cancer patients. Clin Cancer Res. 2005; 11: 5390-5.

15. Rodvold JJ, Mahadevan NR, Zanetti M. Lipocalin 2 in cancer: when good immunity goes bad. Cancer Lett. 2012; 316: 132-8.

16. Candido S, Maestro R, Polesel J, Catania A, Maira F, Signorelli SS, et al. Roles of neutrophil gelatinase-associated lipocalin (NGAL) in human cancer. Oncotarget. 2014; 5: 1576-94.

17. Zhang H, Xu L, Xiao D, Xie J, Zeng H, Wang Z, et al. Upregulation of neutrophil gelatinase-associated lipocalin in oesophageal squamous cell carcinoma: significant correlation with cell differentiation and tumour invasion. J Clin Pathol. 2007; 60: 555-61.

18. Bao G, Clifton M, Hoette TM, Mori K, Deng SX, Qiu A, et al. Iron traffics in circulation bound to a siderocalin (Ngal)-catechol complex. Nat Chem Biol. 2010; 6: 602-9.

19. Chakraborty S, Kaur S, Guha S, Batra SK. The multifaceted roles of neutrophil gelatinase associated lipocalin (NGAL) in inflammation and cancer. Biochim Biophys Acta. 2012; 1826: 129-69.

20. Pacifico F, Pisa L, Mellone S, Cillo M, Lepore A, Leonardi A. NGAL promotes recruitment of tumor infiltrating leukocytes. Oncotarget. 2018; 9: 30761-72

21. Miharada K, Hiroyama T, Sudo K, Danjo I, Nagasawa T, Nakamura Y. Lipocalin 2-mediated growth suppression is evident in human erythroid and monocyte/macrophage lineage cells. J Cell Physiol. 2008; 215: 526-37.

22. Cai L, Rubin J, Han W, Venge P, Xu S. The origin of multiple molecular forms in urine of HNL/NGAL. Clin J Am Soc Nephrol. 2010; 5: 2229-35.

23. Flower DR. The lipocalin protein family: structure and function. Biochem J. 1996; 318: 1-14.

24. Yang J, Moses MA. Lipocalin 2: a multifaceted modulator of human cancer. Cell Cycle. 2009; 8: 2347-52.

25. Cowland JB, Sørensen OE, Sehested M, Borregaard N. Neutrophil gelatinase-

associated lipocalin is up regulated in human epithelial cells by IL-1 beta, but not by TNF-alpha. J Immunol. 2003; 171: 6630-9.

26. Karin M. Nuclear factor-kappaB in cancer development and progression. Nature. 2006; 441: 431-6.

27. Lin WW, Karin M. A citokine-mediated link between innate immunity, inflammation, and cancer. J Clin Invest. 2007; 117: 1175-83.

28. Lu X, Kang Y. Hypoxia and hypoxia-inducible factors: master regulators of metastasis. Clin Cancer Res. 2010; 16: 5928-35.

29. Vaupel P, Mayer A. Hypoxia in cancer: significance and impact on clinical outcome. Cancer Metastasis Rev. 2007; 26: 225-39.

30. Rohwer N, Cramer T. Hypoxia-mediated drug resistance: novel insights on the functional interaction of HIFs and cell death pathways. Drug Resist Updat. 2011; 14: 191-201.

31. Nakamura 1, Hama S, Itakura S, Takasaki I, Nishi T, Tabuchi Y, et al. Lipocalin2 as a plasma marker for tumors with hypoxic regions. Sci Rep. 2014; 4: 7235

32. Yang J, Mcneish B, Butterfield C, Moses MA. Lipocalin 2 is a novel regulator of angiogenesis in human breast cancer. Faseb J. 2013; 27: 45-50.

33. Ariazi EA, Taylor JC, Black MA, Nicolas E, Slifker MJ, Azzam DJ, et al. A new role for ERa: silencing via DNA methylation of basal, stem cell, and EMT genes. Mol Cancer Res. 2017; 15: 152-164.

34. Li SH, Hawthorne VS, Neal CL, Sanghera S, Xu J, Yang J, et al. Upregulation of neutrophil gelatinase-associated lipocalin by ErbB2 through nuclear factorkappaB activation. Cancer Res. 2009; 69: 9163-8.

35. Hu C, Yang K, Li M, Huang W, Zhang F, Wang H. Lipocalin 2: a potential therapeutic target for breast cancer metastasis. Onco Targets Ther. 2018; 11: 8099-106.

36. Wenners AS, Mehta K, Loibl S, Park H, Mueller B, Arnold N, et al. Neutrophil gelatinase-associated lipocalin (NGAL) predicts response to neoadjuvant chemotherapy and clinical outcome in primary human breast cancer. PLoS One. 2012; 7: e45826.

37. Venkatesha S, Hanai JI, Seth P, Karumanchi SA, Sukhatme VP. Lipocalin 2 antagonizes the proangiogenic action of ras in transformed cells. Mol Cancer Res. 2006; 4: 821-9.

38. Zhang $\mathrm{M}$, Huang XZ, Song $\mathrm{YX}$, Gao P, Sun JX, Wang ZN. High Platelet-to-

Lymphocyte ratio predicts poor prognosis and clinicopathological 
characteristics in patients with breast cancer: A meta-analysis. Biomed Res Int. 2017 ;2017: 9503025.

39. Tjon-Kon-Fat LA, Lundholm M, Schroder M, Wurdinger T, ThellenbergKarlsson C, Widmark A, et al. Platelets harbor prostate cancer biomarkers and the ability to predict therapeutic response to abiraterone in castration resistant patients. Prostate. 2018; 78: 48-53.

40. Stuckey R, Aldridge T, Lim FL, Moore DJ, Tinwell H, Doherty N, et al. Induction of iron homeostasis genes during estrogen-induced uterine growth and differentiation. Mol Cell Endocrinol. 2006; 253: 22-29.

41. Stoesz SP, Friedl A, Haag JD, Lindstrom MJ, Clark GM, Gould MN. Heterogeneous expression of the lipocalin NGAL in primary breast cancers. Int J Cancer. 1998; 79: 565-72. 